BULL. AUSTRAL. MATH. SOC.

VOL. $6(1972), 61-67$.

\title{
A characterization of generalized Hall planes
}

\section{N.L. Johnson}

\begin{abstract}
We prove that a translation plane $\pi$ of odd order is a generalized Hall plane if and only if $\pi$ is derived from a translation plane of semi-translation class 1-3a. Also, a derivable translation plane of even order and class 1-3a derives a generalized Hall plane. We also show that the generalized Hall planes of Kirkpatrick form a subclass of the class of planes derived from the Dickson semifield planes.
\end{abstract}

\section{Introduction and background}

Kirkpatrick [6] defines generalized Hall planes as follows:

A translation plane $\pi$ is a generalized Hall plane if and only if $\pi$ admits a collineation group $G$ which fixes a Baer subplane $\pi_{0}$ pointwise and acts simply transitively on the points of $z_{\infty}-\pi_{0} \cap z_{\infty}$.

Kirkpatrick (Theorem 1, [6]) shows that a generalized Hall plane of odd order admits a coordinatization so that the corresponding quasifield is a right two dimensional vector space over $\mathrm{GF}(q), q$ a prime power, where GF $(q)$ coordinatizes $\pi_{0}$. Furthermore, Kirkpatrick defines a class of quasifields that coordinatize generalized Hall planes of odd order and which properly contains the Hall quasifields.

Originally the Hall planes were defined by constructing a

Received 23 August 1971. This work was supported by a grant from the National Science Foundation of the USA. 
coordinatizing quasifield (see [2], pp. 364-365). Albert [1] has shown the finite Hall planes to be precisely the planes derived from the desarguesian planes of square order.

We show analogously that a translation plane $\pi$ of odd order is a generalized Hall plane if and only if $\pi$ is derivable from a translation plane of semi-translation class 1-3a. Furthermore, if the generalized Hall planes of even order can be coordinatized by a quasifield which is a right vector space over $G F(q)$ a similar result holds.

The author [5] considers translation planes derived from semifield planes. We show that the planes of Kirkpatrick (see [6], Section 3) are among the planes obtained by deriving the Dickson semifield planes. The results used in the following sections will be listed for convenience.

We shall write $y=f(x)$ instead of $\{(x, y) \mid y=f(x)\}$ to indicate an affine line. The line at infinity will be denoted by $\tau_{\infty}$ and $x=0 \cap z_{\infty}$ will be denoted by $(\infty)$.

We shall also assume that the reader is somewhat familiar with Ostrom's development of "derivation". We refer the reader to [7] and [8] for the basic background material.

RESULT I (Ostrom [7], Theorem 6). Let $\pi$ be an affine plane of order $q^{2}$ and let $M$ be a set of $q+1$ points on $l_{\infty}$ on $\pi^{*}$ (the projective extension of $\pi$ ). Suppose that for every pair of distinct points $P$ and $Q$ such that $P Q \cap Z_{\infty} \in M$ there exists a projective subplane of $\pi^{*}$ which contains $P, Q$ and $M$. Then the affine parts of the proper subplanes of $\pi^{*}$ which contain $M$ and the affine parts of the lines of $\pi^{*}$ which do not intersect $M$ form the lines of a new affine plane $\bar{\pi}$ (called the plane derived from $\pi$ ) containing the same points as $\pi$.

RESULT II (Ostrom [7], Theorem 7 and Corollary). Let $\sigma$ be a permutation of the points of $\pi$ inducing a collineation of $\pi^{*}$ which carries $M$ into itself. Then $\sigma$ induces a collineation of $\bar{\pi}^{*}$ which carries $\bar{M}$ into itself. Moreover, if $\sigma$ is a translation of $\pi$, then $\sigma$ induces a translation of $\bar{\pi}$.

The term "coordinate system" shall mean a Hall coordinate system (see for example [7], pp. 9-10). 
RESULT III (Ostrom [7] - a strong form of Theorem 9). Let $\pi$ be an affine plane of order $q^{2}$ coordinatized by a system $Q$ such that

(1) $Q$ contains a subfield $F$ of order $q$,

(2) addition is associative and commutative,

(3) $Q$ is a right two dimensional vector space over $F$,

(4) $Q$ is linear with respect to $F$.

Then $\pi$ is derivable and

$\{(x, y) \mid x=a \alpha+c, y=a \beta+b, a \neq 0, b, c$ fixed in $Q$

and for all $\alpha, \beta \in F$ \}

is the set of points of an affine Baer subplane.

RESULT IV (Ostrom [1], Theorem 10). Let $\pi$ be an affine plane coordinatized by a system $Q$ as in Result III and let $t \in Q-F$. Then $\bar{\pi}$ (the plane derived from $\pi$ ) can be coordinatized by a system $\bar{Q}$ such that a point with coordinates $(x, y)=\left(t x_{1}+x_{2}, t y_{1}+y_{2}\right)$ in $Q$; $x_{i}, y_{i} \in F, i=1,2$, has coordinates $(\bar{x}, \bar{y})=\left(t x_{1}+y_{1}, t x_{2}+y_{2}\right)$ in $\bar{Q}$.

Let $(S,+)=\left(G F\left(q^{2}\right),+\right)$. Let $t$ be a fixed element of $S-G F(q)$. The multiplication of the Dickson semifields $(S,+, \cdot)$ is defined as follows:

$t \alpha=t \cdot \alpha, \quad(t \alpha+\beta) \cdot(t \delta+\gamma)=t\left(\alpha \gamma+\beta^{\sigma} \delta\right)+\left(\alpha^{n} \delta^{\rho} g+\beta \gamma\right)$ where $\sigma, n, \rho$ are automorphisms of $\mathrm{GF}(q), g$ a non-square in $\mathrm{GF}(q)$, for all $\alpha, \beta, \delta, \gamma \in \mathrm{GF}(q)$.

It easily follows that the Dickson semifield planes are derivable.

RESULT $V$ (Johnson [5], Theorem $(3.4)(1)$ ). The planes derived from the Dickson semifields may be coordinatized by a right quasifield $(S,+, \star)$ such that $t \star \alpha=t \alpha$,

$$
(t \alpha+\delta) *(t \beta+\gamma)=t\left(\delta-\alpha \beta^{-1} \gamma\right)^{\sigma^{-1}} \beta+\left(\delta-\alpha \beta^{-1} \gamma\right)^{\sigma^{-1}} \gamma+\alpha^{\eta_{\beta}-\sigma \rho} g
$$

for $\beta \neq 0, \alpha, \beta, \delta, \gamma \in G F(q), \delta, \eta, \rho$ automorphisms of $F$, and $g$ a nonsquare in GF $(q)$. Also, $(t \alpha+\delta) * \gamma=t(\alpha \gamma)+\delta \gamma$.

DEFINITION 1.1. Let $\pi$ be a projective plane of order $q^{2}$ and $\pi_{0}$ 
a subplane of order $q$. Let $p$ be a point of $\pi_{0}$ and $L$ a line of $\pi$ such that $L \cap \pi_{0}$ is a line of $\pi_{0}, \pi$ is said to be $\left(p, L, \pi_{0}\right)$-transitive if the stabilizer of $\pi_{0}$ in the group of all $(p, L)$-collineations of $\pi$ induces a collineation group of $\pi_{0}$ such that $\pi_{0}$ is $(p, L)$-transitive (see for example, [3], p. 137).

DEFINITION 1.2. A projective plane $\pi$ is a semi-translation plane with respect to a line $L$ if and only if there is a Baer subplane $\pi_{0}$ containing the line $L$ such that $\pi$ is $\left(p, L, \pi_{0}\right)$-transitive for all points $p \in L \cap \pi_{0}$ (see [3], p. 1372 for another definition).

DEFINITION 1.3. A semi-translation plane $\pi$ with respect to $l_{\infty}$ and subplane $\pi_{0}$ is of class $1-3 a$ if and only if $\pi$ is $\left(p_{\infty}, L, \pi_{0}\right)$-transitive for all lines $L$ of $\pi_{0}$ such that $L I p_{\infty}, p_{\infty}$ a fixed point of $L_{\infty}$ and $\left(p, L, \pi_{0}\right)$-transitive for all points $p I L_{\infty} \cap \pi_{0}$ for all lines of $\pi_{0}$ incident with $p_{\infty}$ (see [3], (2.16), p. 1380$)$.

RESULT VI (Johnson [4], Lemmas (3.1), (3.2)). Let $\pi$ be a semi-translation plane with respect to $\tau_{\infty}$ and Baer subplane $\pi_{0}$. Assume $\pi_{0}$ is coordinatized by $\mathrm{GF}(q)$. If $\pi$ is $\left((0), x=0, \pi_{0}\right)$ - and $\left((\infty), x=0, \pi_{0}\right)$-transitive then $c(\alpha m)=(c \alpha) m$, and $c(\alpha+m)=c \alpha+c m$ for all $c, m$ in a coordinate system for $\pi$ and for all $\alpha \in G F(q)$.

\section{Translation planes of class 1-3a}

A translation plane $\pi$ which contains a Baer subplane $\pi_{0}$ is a semi-translation plane. If $\pi$ is of class 1-3a, $\pi_{0}$ desarguesian, and coordinates are chosen so that $\pi_{0}$ is coordinatized by $\mathrm{GF}(q)$, $p_{\infty}=(\infty)$ (see Definition 1.3), then clearly $\pi$ is $\left((\infty), x=0, \pi_{0}\right)$ - and $\left((0), x=0, \pi_{0}\right)$-transitive. By Result VI and the ordinary properties of a coordinatizing quasifield $Q$ it follows that $Q$ is a right two 
dimensional vector space over GF(q). By Result III, $\pi$ is derivable.

THEOREM 2.1. A translation plane $\pi$ containing a desarguesian Baer subplane of semi-translation class 1-3a is derivable and derives a generalized Hall plane.

Proof. We choose coordinates as above and as in Result IV. By Result III (we also use here the fact that "derivation" is involutory) the $x=0$ of $\pi$ appears as a Baer subplane $\bar{\pi}_{0}$ in the plane $\bar{\pi}$ derived from $\pi$. By Result II, $\bar{\pi}$ is a translation plane and admits a collineation group inherited from the $\left((\infty), x=0, \pi_{0}\right)$ - and $\left((0), x=0, \pi_{0}\right)$-collineation groups of $\pi$.

The group of $\bar{\pi}$ generated by the inherited groups clearly fixes $\bar{\pi}_{0}$ pointwise. Since the original group is simply transitive on the points of $l_{\infty}-\pi_{0} \cap l_{\infty}$ and since the set of lines on these points is not altered by derivation (see Result I) it follows that the inherited group is simply transitive on $\bar{l}_{\infty}-\bar{\pi}_{0} \cap \bar{l}_{\infty}$. Thus, the derived plane $\bar{\pi}$ is a generalized Hall plane.

\section{Generalized Hall planes of odd order}

Let $\bar{\pi}$ be a generalized Hall plane of odd order with Baer subplane $\bar{\pi}_{0}$. By Kirkpatrick's Theorem 1 [6], there is a coordinatizing quasifield $\bar{Q}$ for $\bar{\pi}$ such that $\bar{Q}$ is a right two dimensional vector space over $F=G F(q)$ where $F$ coordinatizes $\bar{\pi}_{0}$.

By Result III, $\bar{\pi}$ is derivable and by Result II the derived plane $\pi$ is a translation plane.

Let $\bar{G}$. denote the group acting simply transitively on $\bar{l}_{\infty}-\bar{\pi}_{0} \cap \bar{l}_{\infty}$. Clearly, $\bar{G}$ induces an automorphism group $\bar{G}_{a}$ on $\bar{Q}$ which fixes $F$ elementwise. Let $\{1, t\}$ be a basis for $Q$ over $F$. Then $\bar{G}_{a}=\left\{\sigma_{\alpha, \beta} ; \alpha \neq 0, \beta \in F\right\}$ where $\sigma_{\alpha, \beta}$ is defined by $t \sigma_{\alpha, \beta}=t \alpha+\beta$.

It follows that the group $\bar{G}$ is generated by the mappings: $\left(t x_{1}+x_{2}, t y_{1}+y_{2}\right) \rightarrow\left(t x_{1} \alpha+x_{2}, t y_{1}^{\alpha+y_{2}}\right)$ and 
$\left(t x_{1}+x_{2}, t y_{1}+y_{2}\right) \rightarrow\left(t x_{1}+x_{2}+x_{1} \beta, t y_{1}+y_{2}+y_{2} \beta\right)$ for all $\alpha \neq 0, \beta \in F$. By Results II and IV, the mappings induce collineations of $\pi$ represented by $\left(t x_{1}+y_{1}, t x_{2}+y_{2}\right) \rightarrow\left(t x_{1} \alpha+y_{1} \alpha, t x_{2}+y_{2}\right)$ and $\left(t x_{1}+y_{1}, t x_{2}+y_{2}\right) \rightarrow\left(t x_{1}+y_{1}, t\left(x_{2}+x_{1} \beta\right)+\left(y_{2}+y_{1} \beta\right)\right)$ or rather $(x, y) \rightarrow(x \alpha, y)$ and $(x, y) \rightarrow(x, x \beta+y)$. These mappings clearly represent $\left((0), x=0, \pi_{0}\right)-$ and $\left((\infty), x=0, \pi_{0}\right)$-transitivity, respectively. Thus, we have the following:

THEOREM 3.1. Generalized Hall planes of odd order are derivable and derive translation planes of semi-translation class 1-3a.

\section{The planes derived from the Dickson semifields}

The known translation planes of class 1-3a are semifield planes. The author has studied planes derived from semifield planes in [5]. In particular, the planes of [5], Theorem (3.1), (1), (3) and (4) are generalized Hall planes.

Kirkpatrick's generalized Hall systems are defined as follows (see Section 3,[6]).

Let $(Q,+)=\left(\mathrm{GF}\left(q^{2}\right),+\right), q$ odd, and $\theta, \varphi$ automorphisms of $\mathrm{GF}(q)$ and $\nu$ a nonsquare of $\mathrm{GF}(q)$.

Define $(z \alpha+\beta) z=z \beta^{\theta}+\alpha^{\varphi} \nu$ for all $z \in Q-G F(q) ; \alpha, \beta \in G F(q)$.

Notice that if $\{I, t\}$ is a basis for $Q$ over $G F(q)$ and $z=t z_{1}+z_{2} ; z_{i} \in G F(q)$, then, for $B \neq 0$,

$$
\begin{aligned}
(t \alpha+\delta)(t \beta+\gamma) & =\left((t \beta+\gamma)\left(\beta^{-1} \alpha\right)+\left(\delta-\beta^{-1} \alpha \gamma\right)\right](t \beta+\gamma) \\
& =(t \beta+\gamma)\left(\delta-\beta^{-1} \alpha \gamma\right)^{\theta}+\left(\beta^{-1} \alpha\right)^{\varphi} \nu \\
& =t\left(\delta-\alpha \beta^{-1} \gamma\right)^{\theta} \beta+\left(\delta-\alpha \beta^{-1} \gamma\right)^{\theta} \gamma+\alpha^{\varphi} \beta^{-\varphi} \nu,
\end{aligned}
$$

which is precisely the *-multiplication with $\theta=\sigma^{-1}, \varphi=n, \varphi \theta=\rho$ of Result V.

Thus, we have the following:

THEOREM 4.1. Kirkpatrick's generalized Hall planes form a proper subclass of the planes derived from the Dickson semifields. 
Note added in proof on 22 September 1971. T.G. Ostrom has pointed out to the author that derivable translation planes of class $1-3 a$ are semifield planes.

\section{References}

[1] A.A. Albert, "The finite planes of Ostrom", Bol. Soc. Mat. Mexicana (2) $11(1966), 1-13$.

[2] Marshall $\mathrm{Hall}, \mathrm{Jr}$, The theory of groups (The Macmillan Company, New York, 1959 ).

[3] Norman Lloyd Johnson, "A classification of semi-translation planes", Canad. J. Math. 21 (1969), 1372-1387.

[4] Norman L. Johnson, "Derivable semi-translation planes", Pacific $J$. Math. 34 (1970), 687-707.

[5] Norman L. Johnson, "Translation planes constructed from semifield planes", Pacific J. Math. 36 (1971), 701-711.

[6] P.B. Kirkpatrick, "Generalization of Hall planes of odd order", BulZ Austral. Math. Soc. 4 (1971), 205-209.

[7] T.G. Ostrom, "Semi-translation planes", Trans. Amer. Math. Soc. 111 (1964), 1-18.

[8] T.G. Ostrom, "Vector spaces and construction of finite projective planes", Arch. Math. 19 (1968), 1-25.

The University of lowa,

lowa City,

lowa,

USA. 